\title{
Denormalization, Smoke-Free Air Policy, and Tobacco Use among Young Adults
}

\author{
Brian C. Kelly \\ Purdue University \\ Mike Vuolo \\ The Ohio State University \\ Laura C. Frizzell \\ The Ohio State University \\ Elaine M. Hernandez \\ Indiana University
}

Key Words: smoking bans; denormalization; smoking; tobacco policy; youth

Word count: 7,990 (including abstract, references, tables, and figures)

\section{Acknowledgements}

This work was supported by the National Institute on Drug Abuse (Grant \#R03DA034933; PI: Vuolo). This research was conducted with restricted access to Bureau of Labor Statistics (BLS) data. The authors would like to thank the staff at the American Nonsmokers' Rights Foundation (ANRF), particularly Maggie Hopkins and Laura Walpert. The views expressed here do not necessarily reflect the views of NIDA, the BLS, or ANRF. We also thank Joy Kadowaki, Emily Harris, Alexandra Marin, Jake Brosius, and Emily Ekl for research assistance. Direct correspondence to Brian C Kelly, Purdue University, Department of Sociology, 700 W State St, West Lafayette, IN 47906 or email bckelly@purdue.edu. 


\section{$\underline{\text { Abstract }}$}

Smoke-free air laws and the denormalization of smoking are important contributors to reductions in smoking during the $21^{\text {st }}$ century. Yet, tobacco policy and denormalization may intersect in numerous ways to affect smoking. We merge data from the National Longitudinal Survey of Youth 1997, Tobacco Use Supplement of the Current Population Survey, American Nonsmokers' Right Foundation, and Census to produce a unique examination of the intersection of smoking bans and denormalization and their influence on any smoking and heavy smoking among young adults. Operationalizing denormalization as complete unacceptability of smoking within nightlife venues, we examine 1) whether smoking bans and denormalization have independent effects on smoking, 2) whether denormalization mediates the influence of smoking bans on smoking, and 3) whether denormalization moderates the impact of smoking bans on smoking. For any smoking, denormalization has a significant independent effect beyond the influence of smoking bans. For heavy smoking, denormalization mediates the relationship between smoking bans and habitual smoking. Denormalization does not moderate the relationship of smoking bans with either pattern of smoking. This research identifies that the intersection of denormalization and smoking bans plays an important role in lowering smoking, yet they remain distinct in their influences. Notably, smoking bans are efficacious even in locales with lower levels of denormalization, particularly for social smoking.

Keywords: smoking bans; denormalization; smoking; tobacco policy; youth 


\section{Denormalization, Smoke-Free Air Policy, and Tobacco Use among Young Adults}

Considerable changes in tobacco policy in recent decades led to dramatic reductions in tobacco use, particularly among young people (Eriksen \& Cerak, 2008). At the same time, wider cultural processes related to stigmatization of tobacco use and denormalization of smoking in public places also contributed to these declines (Bell et al., 2010). Scholarly discussions of smoke-free air laws - colloquially known as smoking bans - often link these policies to processes that denormalize smoking, especially smoking in public, suggesting that policy and denormalization processes are intertwined (Bayer \& Stuber, 2006; Glantz, 1987; Stuber et al., 2008). Yet, little empirical work directly examines the relationship of denormalization to smoking bans and their impact on smoking. We add to the literature on tobacco policy and denormalization by examining whether smoke-free air laws and denormalization of smoking in public have independent or interactive effects on young adult smoking. We merge independent datasets for tobacco policy and an assessment of denormalization with that of a cohort of young adults observed over time, placing those young adults within their wider context of tobacco policy and norms. Further, we measure both policy and denormalization at the lowest geographic level available to incorporate the proximal influence of each, while also accounting for policies that can be passed on multiple levels (i.e. city, county, state). Beyond the specific case of tobacco use, this paper provides evidence for the importance of considering how health policy implementation intersects with cultural processes, such as norm transitions, to produce changes in health behaviors.

Implementation of smoke-free air laws and their impact on smoking 
Smoke-free air laws are successful tools for tobacco control. These policies contributed to reductions of a range of harmful outcomes, including heart attack (Juster et al., 2007; Sargent et al., 2004), respiratory impairment (Eagan et al., 2006; Menzies et al., 2006), and exposure to environmental toxins and particulates (Connolly, 2009; Repace, 2004; Repace et al., 2006). Research also indicates that smoking bans directly affect the prevalence of a range of smoking behaviors (Fitchenburg \& Glantz, 2002; Shang, 2015; Song et al., 2015; Vuolo et al., 2016). Creating a smoke-free environment not only may encourage smokers to quit or reduce consumption, but also prevent the uptake of social smoking among non-smokers - a crucial point for early intervention - and reduce relapse among ex-smokers (Fichtenberg et al., 2002; Lantz 2003). Specifically for young adults, smoke-free air laws have the most significant impact on their smoking behaviors from among a range of possible tobacco control policies (Vuolo et al., 2016).

Although contentious when initially implemented, research has indicated high levels of compliance with smoke-free air laws once in effect (Kelly, 2009; Skeer et al., 2004). Research examining attitudes towards California's smoking ban demonstrated that approval of the policy increased over time, suggesting that smoke-free air laws facilitate a process of normalizing prohibitions and denormalizing smoking (Tang et al., 2003). Although many smokers expressed displeasure with restrictions on smoking in public places, many also stated that they understood the desires of non-smokers to be in smoke-free environments (Bell et al., 2010; Kelly, 2009). Despite efforts by the tobacco industry to disrupt changes (Elias et al., 2018), attitudes towards smoking in public shifted considerably during the period following implementation of smoking bans (Thomson et al., 2009, 2016), including among young people (Johnston et al., 2017). In this manner, scholars have credited smoking bans with changing popular perceptions of the 
acceptability of smoking in public places as well as the act of smoking in general. These cultural shifts in perceptions of smoking behaviors are characterized as denormalization.

\section{Denormalization processes}

Smoking was once considered a fashionable element of social events, but its glamour has disappeared over recent decades (Brandt, 1998). This transformation occurred alongside widespread reductions in smoking among the American public. Denormalization represents a process whereby behaviors once deemed commonplace, acceptable, and ordinary become recast as unacceptable, discredited, and unusual. Norms play an important role in behavior because they establish social monitoring and also become internalized for the self-policing of behavior (Horne, 2003). Through denormalization, the cultural dimensions of how behaviors are constructed within the popular consciousness translate into a vehicle by which social pressures may be marshalled to discourage unhealthy behaviors.

Through denormalization, increases in the unacceptability of smoking play an important role in reducing tobacco use (Alamar \& Glantz, 2006). Public health professionals have come to wield denormalization as a tool for health promotion, particularly for tobacco control. As noted by Colgrove and colleagues, “denormalizing smoking has become a central prong of antitobacco efforts, both as a way of discouraging initiation of smoking and as a means of pressuring current smokers to quit" (2011:2376). Policies, such as advertising restrictions, have been utilized to facilitate denormalization across society. Scholars have also theorized the passage of smoke-free air laws as contributing to the process of denormalization via reductions in the public visibility of smoking as well as the relegation of smoking to spaces separate from the domains of sociability. In this manner, smoking bans may not only directly intervene on smoking by 
restricting where individuals can smoke, they may also subtly signal that the behavior is unacceptable to perform in public. As Glantz (1987:747) described, smoking bans have the potential to reduce smoking within the population because they "undercut the social support network for smoking by implicitly defining smoking as an anti-social act."

By rendering the act of smoking unacceptable in the public domain, smoking bans contribute to changes in social norms surrounding smoking. As social interactions in nonsmoking public domains become routinized, they may encourage reductions or cessation of smoking more generally. In this manner, declining acceptability of smoking in public may spill over to reduce the normative basis of smoking even in private domains. Yet, such processes have raised concerns among scholars about the production of stigma that may harm those who continue to smoke (Bell et al., 2010). For such reasons, the manner in which denormalization unfolds matters for smoking outcomes.

\section{Denormalization, stigma, and place}

Denormalizing smoking may contribute to increases in stigma associated with smoking in public and smoking more generally. Yet, scholars have raised questions about tobacco-related stigma as an acceptable exception in a public health domain in which stigmatization is routinely discouraged (Bayer, 2008; Stuber et al., 2008). As Bayer and Stuber note, "Although such restrictions have been imposed on the act of smoking, they have inevitably had profound impacts on smokers themselves and their social standing" (2006:47). Concerns about the attribution of stigma to smokers mainly center on the effect of stigma on care-seeking, social support, and other social mechanisms by which smoking cessation and related health care behaviors may occur (Stuber et al., 2008). Additionally, it is not simply that others stigmatize smokers, but that 
smokers themselves enact labels of self-stigma as the permissibility of smoking in public declines (Evans-Polce et al., 2015).

While unlikely a panacea for the prevention of stigma, a focus on denormalization of public smoking provides opportunity to intervene on smoking without direct criticism of smokers themselves. The normative basis of behaviors can be detached from their practice in the public domain without stigmatization of the behavior itself. For instance, sexual activity between two consenting adults is considered normative adult behavior, and yet acts of public sexual interaction are decidedly non-normative. The stigma is associated with the public performance of the act, rather than the act itself. Context matters, and if effectively organized, smoke-free air laws hold promise for the denormalization of smoking as a public behavior while not contributing to the ways smokers themselves are stigmatized. As such, investigations of the linkages between smoke-free air laws and denormalization remain important.

Variations in perceptions of smoking in public are also related to the strength of denormalization. Public support for restaurant bans of smoking has generally been higher than restrictions in "adult only" spaces, such as bars and nightclubs (Alamar \& Glantz, 2006). Although concerns about smoking in restaurants and other places relate, in part, to concerns about the exposure of children and adolescents not only to cigarette smoke but also to witnessing the act of smoking, these concerns do not arise for bars and nightclubs, which may explain the discrepancies in support for restaurant bans and bans in nightlife spaces. Thus, support for smoking bans within venues such as bars indicates a stronger denormalization of smoking in public, as these considerations do not directly relate to exposure of minors to smoking.

Independent or Interactive Effects of Smoke-Free Air Policy and Denormalization 
The scientific literature has largely considered smoke-free air laws and denormalization to occur hand in hand (Bayer \& Stuber, 2006; Glantz, 1987). Yet, empirical examinations of the linkages between smoking bans, denormalization, and tobacco use are lacking, and how they interrelate is often unclear. The relationship between smoking bans and denormalization may not be singular in nature. Rather, as described below, there may be several ways in which smokefree air policies and denormalization intersect in their effects on smoking.

The denormalization of public smoking, as a wider cultural phenomenon, may have an independent effect from that of smoke-free air laws. The denormalization of smoking in public places is a broader cultural process not simply limited to the spaces in which smoking bans apply. As noted above, perceived unacceptability of smoking within "adult only" spaces indicates the strongest form of denormalization of public smoking. Yet, reductions in the acceptability of public smoking may influence smoking behaviors beyond the effect of smoking ban implementation. Given that behavioral norms become strengthened through the social practices that play out in public contexts, the absence of smoking within these venues may enhance denormalization, while the wider cultural process of denormalization concurrently retains an effect beyond that shaped by the implementation of smoking bans. As such, denormalization may operate independently, but in parallel with the effects of smoking bans, leading to the following hypothesis.

Hypothesis 1: Denormalization reduces smoking beyond the effect produced by smoking bans.

Denormalization also may be the key mechanism by which smoking bans work to reduce smoking within the population. Studies have identified that restrictions on smoking indoors 
influence the social acceptability of smoking (Albers et al., 2004; Brown et al., 2009). Such denormalization borne out of policy implementation may shape patterns of smoking within the population. That is to say, the erosion of norms conducive to smoking may directly result from the implementation of a smoking ban, and in turn the erosion of such norms leads to reductions in smoking within the population. Thus, denormalization may be a critical mediating mechanism by which smoking bans impact population-level smoking. As described above, this process has been widely theorized within the literature (Bayer \& Stuber, 2006).

Hypothesis 2: Denormalization mediates the relationship between smoking bans and smoking.

Lastly, smoke-free air laws may have a greater impact when implemented in locations with high levels of denormalization. Policies do not necessarily affect all individuals in the same manner. For example, Boardman and colleagues (2010) identified that genetic influences on smoking matter within the context of a changing policy environment. Although not very well studied, the local normative context may also play a role in shaping the impact of health policy implementation (Kinzig et al., 2013), and for tobacco in particular, there is likely considerable interplay between policy, norms, and behavior that develops over time (Green et al., 2006). More specifically, smoking bans may be more effective in locations where there is stronger denormalization (i.e. lower support for public smoking). The wider cultural process of denormalization may thus help determine the efficacy of smoking bans as "upstream interventions." This moderating mechanism is less well conceptualized within the literature, yet remains critical with respect to deciphering whether attitudes within locations of implementation matter for the influence of such policies. 
Hypothesis 3: Smoking bans will have greater effects on smoking within locales that exhibit greater denormalization of smoking in public places.

Within considerations of how smoking bans and denormalization intersect, drawing distinctions between types of smoking behaviors remains important. Specifically, denormalization may affect social smokers differently than habitual smokers. This is noteworthy given that social smoking has remained common even though daily smoking has declined considerably (Schane et al., 2009). Habitual smokers may be less susceptible to the influence of either smoke-free policies or denormalization because of both physiological dependence and the habituation of behavioral routines. As such, examining the effects of these processes on any smoking and heavy smoking remains essential to decipher the ways that smoke-free air policies and denormalization intersect.

In light of the need for empirical study of the relationship between smoke-free air laws and denormalization, we examine how both influence smoking among young adults. Employing random-effects hierarchical models, we utilize a nationally representative sample of young people who came of age during a period of significant policy implementation and denormalization of smoking, as well as data independent of these young people to measure tobacco control policy and denormalization of public smoking. The results provide important information about the intersection of smoking bans and denormalization in relation to reducing smoking during a period of the life course when intervention makes a high impact on long-term health and well-being. 


\section{METHODS}

Individual-Level Data: National Longitudinal Survey of Youth 1997 (NLSY97)

Individual-level data come from the NLSY97, a nationally representative, geocoded sample $(N=8,984)$ designed to track the transition of youth into adulthood. Adolescents (12-16) were randomly sampled in 1997 and surveyed annually. The retention rate was nearly $83 \%$ in 2011. The restricted-access, geocoded NLSY97 identifies the respondents' core-based statistical area (CBSA; i.e. metropolitan/micropolitan area), county, and state. We analyzed a subset of respondents whose city of residence could be identified by combining CBSA and county information with a variable assessing whether the respondent lived in a principal city within the metro area. Thus, we restrict our analyses to those living in the largest principal city of a CBSA, given the importance of the local level within a multilevel policy context. We also restrict analyses to waves 2004 and later (ages 19-31), as this was the first year in which CBSA data is available. Before 2004, only metropolitan statistical area (MSA) was available. Though it would increase the number of time-points, using MSA $(>50,000$ people) rather than CBSA $(>10,000$ people) would reduce the number of cities (and respondents) analyzed. Given our focus on local policy and denormalization, we prioritized adding more cities over more time-points, while expanding the included cities to a lower population threshold, such that not only large urban areas are included. We restricted analyses to age 19 and older, which constitutes an age when many young adults begin to frequent venues, such as nightclubs and bars, with the most between-city variation in smoke-free air policy. In the NLSY97, 4,341 respondents are in this subset, and they are similar to the wider sample on individual-level variables, with two exceptions. Blacks are somewhat overrepresented, with Whites underrepresented. The subset is also more likely to work. 
Dependent Variables. Each year, respondents who indicated they ever smoked an entire cigarette were asked the number of days they smoked during the past 30 days, and the number of cigarettes they smoked each day on those 30 days. We created two outcome variables: one indicating any past 30-day cigarette use and a second for those who reported smoking at least a pack per day. Pooled across years, $32.8 \%$ reported smoking during the past 30 days, while $4.6 \%$ reported smoking at least a pack daily. Table 1 shows descriptive statistics for all variables.

[Table 1]

Independent Variables. We included a considerable battery of control variables related to tobacco use. Given that age is central to patterns of substance use, we chose age as our time metric (Yang, 2010), including a quadratic term as this fit the data better than any other polynomial. Age in 1997 controls for cohort effects. Since youth who initiate smoking as adolescents have higher odds of smoking, especially heavy smoking, as adults (Wills et al. 2004), we controlled for past 30 day smoking at age 17. Regarding family, we included timevarying indicator variables for whether respondents lived with a parent, were married, and had children (Brown \& Rinelli, 2010; Fleming et al., 2010). We accounted for residential migration via a dummy variable for a past year move across at least one county. For work factors, we included time-varying categorical variables for job status and job schedule (Johnson, 2004). To assess peer influences, we included the proportion of peers who smoked at baseline (Alexander et al., 2001). For academic performance, we included a dummy variable for receiving "mostly A's" in high school (Schulenberg et al., 1994). To control for mental health (Swan et al., 2004), we included a five-item scale for depression (alpha $=0.77$ ). To account for intergenerational 
health influences (Kandel et al., 2015), we included parents' self-reported health. We included several measures for socioeconomic status. We measured SES of household of origin by respondent-reported parents' education level (Soteriades \& DiFranza, 2003; Pampel et al., 2014). Respondents' SES were assessed by a time-varying measure that combined school enrollment status and degree attainment (Pampel et al., 2014; Schulenberg et al., 1994). Finally, we included controls for race/ethnicity (Johnson \& Hoffman, 2000; Pampel, 2008), U.S. nativity, and gender (Pampel, 2001).

\section{Policy Data: American Nonsmokers' Rights Foundation (ANRF)}

Policy data come from the restricted ANRF tobacco policy database. ANRF collected a complete national repository of tobacco-related ordinances and regulations across the country. The main policy variable assesses whether the respondent lived in a locale with a comprehensive smoking ban, which mandate that workplaces, bars, and restaurants are $100 \%$ smoke-free. We created a location-year dataset at the state-, county- and city-level for each data year. Since state and county policies are not independent of city policy (e.g., a state policy automatically means a city-level ban, and therefore, must match), we recoded cities within states and counties with bans (without city exceptions) to reflect this status. We used FIPS codes to link the geocoded NLSY97 to ANRF data, establishing the tobacco policy context where respondents resided. We used this procedure to include additional policies as controls to incorporate the main domains of tobacco control (Friend et al., 2011), including youth possession restrictions, single cigarette sales restrictions, complete vending machine prohibitions, advertising restrictions, and excise taxes. 
Denormalization Data: Tobacco Use Supplement, Current Population Survey (TUS-CPS)

The TUS-CPS is a nationally representative sample with data on current and former use of tobacco products, workplace smoking policies, and personal attitudes about tobacco use. It has been intermittently collected from 1992 to 2015 , and the provided weights were used to ensure correct measurement at the aggregate level. We use the years overlapping with our NLSY97 subsample, linearly interpolating years between data collections. Importantly, this data provides an independent measure of denormalization in the wider metro area untied to perceptions of youth in the NLSY97.

Given that the policy of interest is a comprehensive smoking ban, we use a question in the TUS-CPS that mirrors this policy. The question asked respondents to provide their personal opinion about whether smoking should be allowed in nightlife venues ("Inside bars, cocktail lounges, and clubs, do you think that smoking should be allowed in all areas, allowed in some areas, or not allowed at all?"). Response options were: allowed in all areas, allowed in some areas, and not allowed at all. Bar bans represent the most restrictive form of smoking bans, relative to questions about workplaces and restaurants. The last response option then represents complete unacceptability of smoking indoors in public spaces, matching our comprehensive smoking ban defined as locations with no exceptions for smoking in workplaces, restaurants, or bars. Across all years, there is almost no missing data, with only $3.2 \%$ of participants not responding. Our aggregated version of this measure is thus based on the responses of 335,777 individuals. We aggregated this measure to the lowest possible geographic level for the TUSCPS that coincided with a geocode in the NLSY97, the metro area. Some metro areas in the NLSY97 do not appear in the TUS-CPS, such that the match is not perfect. There was, however, substantial overlap between these two nationally representative datasets. Relative to our total 
subsample described above, our final NLSY97 analytic sample totals 15,588 datapoints (79.3\% of possible matches) across 3,855 respondents (88.8\%) in 223 matched metros.

In terms of measurement, we created a binary version of the opinion question by coding "not allowed at all" as 1 and allowed in all or some areas as 0 since the former represents complete unacceptability of smoking indoors in public spaces, matching our comprehensive smoking ban measure. Our measure of denormalization then is the average of this binary at the metro-level in the percentage scale. That is, it represents the percentage of metro residents who believe that smoking is totally unacceptable in indoor public spaces, or denormalization of such behavior. Although not shown, we alternatively used the average of the 3 point ordinal scale to measure denormalization, and all conclusions reported below remained the same with this robustness check. We use the average of the binary version because it better taps into complete unacceptability of indoor public smoking, better measuring denormalization, while also having a more intuitive scale (i.e. percentage points).

\section{City-Level Controls: U.S. Census}

Several city-level measures from Census data are included as controls (Mathur et al., 2013). To include both population size and density, we created a categorical measure of population, while density is continuous (logged due to skewness). We included the percentage of female-headed households, a proxy for other measures such as poverty and income (LeClere et al., 1998). To measure ties to the community, we used percentage of owner-occupied housing. Finally, we included percentage of non-Hispanic whites and percentage of minors to account for community racial and age composition, respectively. 


\section{Methods}

Given the various levels of analysis and a binary outcome, we use multilevel logistic regression models, also known as mixed effects models, to estimate the effects of policy and denormalization on young adult smoking. In our analysis, observations are nested within individuals, who are nested within cities. Individuals can move across cities, such that the data is more akin to a cross-classified multilevel model where observations are cross-classified by both individual and city. Among our analytic sample, only 18\% were located in more than one city. Thus, the cross-classification of individual and city represents a very sparse, large matrix. In such cases, the loss in precision of the estimation of the variance components from using the typical multilevel structure is slight relative to the great computational advantage (Goldstein, 1999). For longitudinal datasets, this will often be the case given that individuals move across a limited number of geographic units relative to the total number of geographic units. With $82 \%$ of respondents having lived in only one city, there is no cross-classification for such individuals. We also included a fixed-effect to adjust for the average effect of moving across geographic units since the last survey.

Our three level model thus includes random intercepts for both individual-level (Level 2) and city-level (Level 3). These models adjust for person- and city-level averages through a variance parameter defining a normal distribution for each of those averages. At the lowest level denoting time (Level 1), the predictors represent time-varying measures. At the individual-level, we have the time-invariant characteristics of the respondent. Since policy, denormalization, and city characteristics time-vary, they are actually Level 1 variables. As we do not include static city characteristics, the random intercept is the only term at Level 3. All models were fit in Stata 14.2 
using the "xtmelogit" procedure. Indicators of multicollinearity were well within acceptable bounds (mean VIF $=2.22$; highest $\mathrm{VIF}=5.40$ ).

\section{RESULTS}

Figure 1 depicts the growth across years in both comprehensive smoke-free air policies and denormalization of indoor smoking for locations where the NLSY97 respondents resided. The percentage of respondents living in a city subject to a comprehensive smoking ban increased dramatically from $14.9 \%$ in 2004 to $58.7 \%$ in 2011 . Denormalization of indoor public smoking for the metro in which the NLSY97 resides also increases, with the average percentage of metro residents indicating smoking in bars is not at all acceptable increasing from 36.8\% in 2004 to $53.3 \%$ in 2011 . Thus, initially, the average unacceptability of indoor smoking was actually higher than the percentage of respondents who resided in a location with a comprehensive smoking ban, but policy implementation eclipsed public support for comprehensive bans over the course of the period of observation. Policy implementation took time to catch up with changing public opinion, in part due to opposition from tobacco industry lobbying efforts (Elias et al., 2018). Perhaps surprisingly, denormalization does not keep pace with smoking ban implementation. We would expect that as more locations pass comprehensive bans that include bars, the corresponding measure of unacceptability of smoking in bars would at least maintain equivalence. But this is not the case. This provides initial evidence that policies and norms of unacceptability may not be directly correlated. In fact, in 2011, the average percentage agreeing that smoking in bars is never acceptable was $55.7 \%$ in locations that had enacted a comprehensive ban by that point and $49.3 \%$ for those who did not, a rather small magnitude difference. We do not discuss this trend over time since many cities enact bans over this period, 
thus switching categories. Examining these differences over time creates non-mutually exclusive category membership.

[Figure 1]

Next we consider how a policy that restricts smoking behavior and the corresponding measure of denormalization affects smoking, shown in Table 2. To focus on our variables of interest, we do not show control variables. Full models are available in Appendix A and B. We begin with models for any past month tobacco smoking in the top panel of Table 2 . Model 1 shows a significant negative effect of living in a city subject to a comprehensive smoking ban, whereby the odds of any smoking are $46.6 \%$ lower in cities with a ban $(p<.001)$. Model 2 adds the measure of denormalization of indoor public smoking, which is also significant. For a more meaningful scale, the tables and results use an increase of 10 percentage points (we note this is close to a standard deviation for this variable, 13.25). A 10 percentage point increase in smoking being never acceptable in bars decreases the odds of any smoking by $16.2 \%(p<.05)$. The comprehensive smoking ban effect remains significant, although it is attenuated by denormalization by about $30.4 \%$ (in the linear scale). In model 3, we consider these effects with a robust battery of controls for the policy, city, and individual. While the controls reduce the magnitude of both measures, they remain significant $(p<.05)$. The odds of any smoking is $24.4 \%$ lower for those residing in cities with a smoking ban. For a 10 percentage point increase in denormalization, the odds of any smoking decrease by $10.5 \%$. Thus, both policy and denormalization affect the odds of any smoking in the past month.

[Table 2]

Next, we consider heavy smoking, specifically smoking a pack per day over the past month (lower panel of Table 2). Beginning again with the bivariate model, we find that the odds 
of smoking a pack per day are $42.8 \%$ lower among respondents in cities with a comprehensive smoking ban $(p<.01)$. When we add denormalization to the model, however, the effect of the ban becomes non-significant. For a 10 percentage point increase in denormalization of indoor public smoking, the odds of smoking a pack per day decrease by $22.4 \%(p<.01)$. In the full model with controls, this pattern of results holds, with the same coefficient for denormalization, such that the controls do not explain the relationship between denormalization and smoking a pack per day.

Finally, in models 4 and 5, we consider the interaction between policy and denormalization for both outcomes. In these models, the coefficient for denormalization represents the slope for respondents in cities with no smoking ban, while the coefficient for the interaction shows whether the slope of denormalization for respondents in cities with a ban is different. For both outcomes, the results are similar: we find no evidence for an interactive effect, regardless of the inclusion of control variables. From this empirical evidence, we can conclude that the effect of residing in a location with a ban is not dependent upon the surrounding level of denormalization.

We considered several robustness checks. First, we split smoking bans into three categories: comprehensive smoking ban, non-comprehensive ban, and no restrictions. The latter two groups recode what was formally zeroes into two even groups. The difference between noncomprehensive versus no restrictions was not significant across the models, statistically justifying our original coding choice and maintaining consistency between our policy measure and the denormalization measure (see Appendix C). Second, we reran our models for daily pack smoking with only those reporting lifetime smoking to eliminate never smokers who are less likely at risk of progressing to pack smoking. This subset retains $73.6 \%$ of our original analytic sample. As Appendix D shows, the results were nearly identical. 


\section{DISCUSSION}

The tobacco control literature positions smoke-free air laws and denormalization as important contributors to reductions in smoking during the $21^{\text {st }}$ century (Albers et al., 2004; Brown et al., 2009; Glantz, 1987). As denormalization unfolds, increasing unacceptability of a behavior is rooted in cultural shifts that inform social norms, which may interrelate with policy changes. In the analyses presented above, we assessed the intersection of tobacco policy and denormalization by examining whether smoke-free air laws and denormalization of smoking in public - operationalized as complete unacceptability of smoking within nightlife venues - have independent or interactive effects on smoking. By bringing together several datasets, we utilized measures of denormalization and policy independent of our sample of young people observed. Overall, our findings indicate that smoke-free indoor air laws are directly connected to the process of denormalization, but also operate independently in some ways. Yet, how they come together may influence social smoking and heavy smoking differently. We specified three hypotheses concerning the intersection of smoking bans and denormalization; these accounted for independent, mediating, and moderating effects.

Starting with the first hypothesis, we examined whether denormalization reduced smoking beyond the effect produced by smoking bans. We found support for this hypothesis for any smoking. We identified that for any smoking, smoking bans and denormalization have simultaneous effects that reduce the likelihood of engaging in smoking at all in the past month. In this manner, denormalization has an effect on any smoking beyond that produced by smoking ban implementation. This provides evidence that smoking bans' influence on any smoking is independent of the wider cultural process of denormalization of public smoking. In contrast, 
smoking bans do not affect heavy smoking independent of denormalization. Generally, these results provide further evidence that denormalization plays an important role in reducing smoking alongside policy.

The second hypothesis indicated that denormalization would mediate the relationship between implementation of a smoking ban and smoking behaviors. We found support for this hypothesis for both smoking outcomes. The introduction of denormalization into the models for any smoking considerably reduced the magnitude of the effect of smoking bans, although the effect of smoking bans remains significant. This provides some support that denormalization partially explains the effect of smoking bans on any smoking among young adults, although both remain important. For those who smoke at least a pack per day, the effect of smoking ban implementation is considerably reduced in magnitude and becomes statistically non-significant. This indicates that smoking bans likely influence heavy smoking via the process of denormalization. As such, we can consider that denormalization is a key mechanism by which smoking bans affect smoking among young people, particularly for heavy smoking. In this manner, we contend that smoking ban implementation can affect the prevalence of smoking within the population by rendering the act of smoking in public as inappropriate and atypical, as has been widely theorized in the literature (Bayer \& Stuber, 2006; Glantz, 1987)

Finally, our third hypothesis indicated that smoking bans would have a stronger effect within cities that exhibit greater denormalization of smoking in public places. We found no evidence for this hypothesis. In this manner, the wider cultural components of denormalization do not reinforce the effects of smoking bans and do not make them more effective in locales with strong norms against public smoking. Yet, this also holds promise for the utility of smoking bans in tobacco control efforts, as the wider cultural context of denormalization does not matter for 
smoking bans to have an effect. Smoking bans remain highly effective for reducing any smoking among young adults, even in locales with weak norms against public smoking. This is particularly noteworthy with respect to the possibility of effecting changes in youth smoking in locales where people do not view public smoking as a concern.

Overall, these findings highlight the importance of both smoking bans and the process of denormalization in shaping tobacco use among young adults. This research identifies opportunities to intervene on smoking during a particularly important period of the life course, when substance use peaks and behavioral patterns of substance use become routinized in ways that shape later life health outcomes and longevity (Chen \& Jacques-Tiura, 2014; Fenelon \& Preston, 2012; Pampel, 2006). These results further contribute to a literature that has identified smoking bans and denormalization as important tools in tobacco control, especially among young people. Yet, currently, state-wide comprehensive smoke-free air policies that prohibit smoking in nightlife venues have been adopted by only approximately half of U.S. states, indicating that opportunities for policy intervention remain considerable. Future research should also more fully identify how denormalization set the political stage for smoking ban adoption.

\section{Limitations}

While this study has many strengths, we must note certain limitations. First, we only included young adults whose location we could identify as a principal city, restricting analyses to respondents who resided in central cities of CBSAs. Although the analyses provide important information about the intersecting role of smoking bans and denormalization on young adult smoking, we are careful to limit our generalizability to young people in such locales. In particular, we lack information on the effect of policy and denormalization on young people in 
rural areas. We encourage future research in this area because local bans are less likely in rural areas, which may affect these processes in distinct ways. Second, we cannot contend that these processes influence smoking cessation among long-term smokers, another key element of tobacco control strategies, but encourage future research on this topic. Further, we are unable to assess neighborhood-level norms towards smoking, which have been shown to be especially important for young people (Glenn et al., 2017). Lastly, our measure of denormalization encompasses the metro area rather than the city, but this provides the opportunity to capture a wider cultural trend in denormalization in that locale, and the ability to bring these independent datasets together in a test of denormalization and policy is a major strength. We also note that there may be alternative strategies to operationalize denormalization beyond that measured by unacceptability in bars.

\section{Conclusions}

Denormalization remains a key component of reductions in smoking across the population, and has links to the implementation of smoke-free indoor air laws. For social smoking, denormalization has independent effects beyond the effect of smoking bans, and also partially mediates the influence of bans on any smoking among young adults. For heavy smoking, denormalization of public smoking is a significant mediating mechanism for the prevention of heavy smoking among young people. Smoking bans remain effective even in locales with weak norms against public smoking. From evidence that uniquely linked multiple independent datasets, these findings provide further evidence that smoking bans are an effective tool for policymakers interested in intervening on youth smoking in the interests of health promotion. Beyond tobacco use specifically, these findings highlight the importance of 
considering how policy implementation intersects with cultural processes, such as norm transitions, to produce changes in health behaviors in meaningful ways. 


\section{REFERENCES}

Alamar, B., Glantz, S.A., 2006. Effect of increased social unacceptability of cigarette smoking on reduction in cigarette consumption. American Journal of Public Health, 96, 13591363.

Albers, A.B., Siegel, M., Cheng, D.M., Biener, L., Rigotti, N.A., 2004. Relation between local restaurant smoking regulations and attitudes towards the prevalence and social acceptability of smoking: a study of youths and adults who eat out predominantly at restaurants in their town. Tobacco Control, 13, 347-355.

Alexander, C., Piazza, M., Mekos, D., Valente, T., 2001. Peers, schools, and adolescent cigarette smoking. Journal of Adolescent Health, 29, 22-30.

Bayer, R., 2008. Stigma and the ethics of public health: not can we but should we. Social Science \& Medicine, 67, 463-472.

Bayer, R., Stuber, J., 2006. Tobacco control, stigma, and public health: rethinking the relations. American Journal of Public Health, 96, 47-50.

Bell, K., Salmon, A., Bowers, M., Bell, J., McCullough, L., 2010. Smoking, stigma and tobacco 'denormalization': further reflections on the use of stigma as a public health tool. Social Science \& Medicine, 70, 795-799.

Brandt, A.M., 1998. Blow some my way: passive smoking, risk, and American culture. Pp. 164187, in Lock, S., Reynolds, L., Tansey, E.M.(Eds.) Ashes to Ashes: The History of Smoking and Health. Amsterdam: Rodopi.

Brown, A., Moodie, C., Hastings, G., 2009. A longitudinal study of policy effect (smoke-free legislation) on smoking norms: ITC Scotland/United Kingdom. Nicotine \& Tobacco Research, 11, 924-932. 
Brown, S.L., Rinelli, L.N., 2010. Family structure, family processes, and adolescent smoking and drinking. Journal of Research on Adolescence, 20, 259-273.

Chen, X., Jacques-Tiura, A.J., 2014. Smoking initiation associated with specific periods in the life course from birth to young adulthood: data from the National Longitudinal Survey of Youth 1997. American Journal of Public Health, 104, e119-e126.

Colgrove, J., Bayer, R., Bachynski, K. E., 2011. Nowhere left to hide? The banishment of smoking from public spaces. New England Journal of Medicine, 364, 2375-2377.

Connolly, G.N., Carpenter, C.M., Travers, M.J., Cummings, K.M., Hyland, A., Mulcahy, M., Clancy, L., 2009. How smoke-free laws improve air quality: a global study of Irish pubs. Nicotine \& Tobacco Research, 11, 600-605.

Eagan, T.M.L., Hetland, J., Aarø, L.E., 2006. Decline in respiratory symptoms in service workers five months after a public smoking ban. Tobacco Control, 15, 242-246.

Elias, J., Hendlin, Y.H., Ling, P.M. 2018. Public versus internal conceptions of addiction: An analysis of internal Philip Morris documents. PLoS Medicine, 15, e1002562.

Eriksen, M.P., Cerak, R.L., 2008. The diffusion and impact of clean indoor air laws. Annual Review of Public Health, 29, 171-185.

Evans-Polce, R.J., Castaldelli-Maia, J.M., Schomerus, G., Evans-Lacko, S.E., 2015. The downside of tobacco control? Smoking and self-stigma: a systematic review. Social Science \& Medicine, 145, 26-34.

Fenelon, A., Preston, S.H., 2012. Estimating smoking-attributable mortality in the Unites States. Demography, 49, 797-818.

Fichtenberg, C.M., Glantz, S.A., 2002. Effect of smoke-free workplaces on smoking behaviour: systematic review. BMJ, 325, 188-191. 
Fleming, C.B., White, H.R., Catalano, R.F., 2010. Romantic relationships and substance use in early adulthood: an examination of the influences of relationship type, partner substance use, and relationship quality. Journal of Health and Social Behavior, 51, 153-167.

Friend, K.B., Lipperman-Kreda, S., Grube, J.W., 2011. The impact of local U.S. tobacco policies on youth tobacco use: a critical review. Open Journal of Preventive Medicine, 1, 34-43.

Glantz, S.A., 1987. Achieving a smokefree society. Circulation, 76, 746-752.

Glenn, N.M., Lapalme, J., McCready, G., Frohlich, K.L., 2017. Young adults’ experiences of neighbourhood smoking-related norms and practices: a qualitative study exploring placebased social inequalities in smoking. Social Science \& Medicine, 189, 17-24.

Goldstein, H., 1999. Multilevel Statistical Models, First Internet Edition. London: Institute of Education.

Green, L.W., Orleans, C.T., Ottoson, J.M., Cameron, R., Pierce, J.P. Bettinghaus, E.P., 2006. Inferring strategies for disseminating physical activity policies, programs, and practices from the successes of tobacco control. American Journal of Preventive Medicine, 31, 6681.

Horne, C. 2003. The internal enforcement of norms. European Sociological Review, 19, 335343.

Johnson, M.K., 2004. Further evidence on adolescent employment and substance use: differences by race and ethnicity. Journal of Health and Social Behavior, 45, 187-197.

Johnson, R.A., Hoffmann, J.P. 2000. Adolescent cigarette smoking in U.S. racial/ethnic subgroups: findings from the National Education Longitudinal Study. Journal of Health and Social Behavior, 41, 392-407. 
Johnston, L.D., O’Malley, P.M., Miech, R.A., Bachman, J.G., Schulenberg, J.E., 2017. Monitoring the Future national survey results on drug use, 1975-2016: Overview, key findings on adolescent drug use. Ann Arbor: Institute for Social Research, University of Michigan.

Juster, H.R., Loomis, B.R., Hinman, T.M., Farrelly, M.C., Hyland, A., Bauer, U.E., Birkhead, G.S., 2007. Declines in hospital admissions for acute myocardial infarction in New York state after implementation of a comprehensive smoking ban. American Journal of Public Health, 97, 2035-2039.

Kandel, D.B., Giesler, P.C., Mei-Chen, H., 2015. Intergenerational patterns of smoking and nicotine dependence among US adolescents. American Journal of Public Health, 105, e63-72.

Kelly, B.C., 2009. Smoke-free air policy: subcultural shifts and secondary health effects among NYC club-going youth. Sociology of Health \& Illness, 31, 569-582.

Kinzig, A.P., Ehrlich, P.R., Alston, L.J., Arrow, K., Barrett, S., Buchman, T.G., Daily, G.C., Levin, B., Levin, S., Oppenheimer, M. Ostrom, E., 2013. Social norms and global environmental challenges: the complex interaction of behaviors, values, and policy. BioScience, 63, 164-175.

Lantz, P.M., 2003. Smoking on the rise among young adults: implications for research and policy. Tobacco Control, 12, i60-i70.

Leclere, F.B., Rogers, R.G., Peters, K., 1998. Neighborhood social context and racial differences in women's heart disease mortality. Journal of Health and Social Behavior, 39, 91-107. 
Mathur, C., Erickson, D.J., Stigler, M.H., Forster, J.L., Finnegan Jr, J.R., 2013. Individual and neighborhood socioeconomic status effects on adolescent smoking: a multilevel cohortsequential latent growth analysis. American Journal of Public Health, 103, 543-548.

Menzies, D., Nair, A., Williamson, P.A., Schembri, S., Al-Khairalla, M.Z., Barnes, M., Fardon, T.C., McFarlane, L., Magee, G.J., Lipworth, B.J., 2006. Respiratory symptoms, pulmonary function, and markers of inflammation among bar workers before and after a legislative ban on smoking in public places. JAMA, 296, 1742-1748.

Pampel, F.C., 2001. Cigarette diffusion and sex differences in smoking. Journal of Health and Social Behavior, 42, 388-404.

Pampel, F.C., 2006. Socioeconomic distinction, cultural tastes and cigarette smoking. Social Science Quarterly, 87, 19-35.

Pampel, F.C., 2008. Racial convergence in cigarette use from adolescence to the mid-thirties. Journal of Health and Social Behavior, 49, 484-498.

Pampel, F.C., Mollborn, S., Lawrence, E.M., 2014. Life course transitions in early adulthood and SES disparities in tobacco use. Social Science Research, 43, 45-59.

Repace, J.L., 2004. Respirable particles and carcinogens in the air of Delaware hospitality venues before and after a smoking ban. Journal of Occupational and Environmental Medicine, 46, 887-905.

Repace, J.L., Hyde, J.N., Brugge, D., 2006. Air pollution in Boston bars before and after a smoking ban. BMC Public Health, 6, 266.

Sargent, R.P., Shepard, R.M., Glantz, S.A., 2004. Reduced incidence of admissions for myocardial infarction associated with public smoking ban: before and after the study. British Medical Journal, 328, 977-980. 
Schane, R.E., Glantz, S.A., Ling, P.M., 2009. Nondaily and social smoking: an increasingly prevalent pattern. Archives of Internal Medicine, 169, 1742-1744.

Schulenberg, J.E., Bachman, J.G., O’Malley, P.M., Johnston, L.D., 1994. High school educational success and subsequent substance use: a panel analysis following adolescents into young adulthood. Journal of Health and Social Behavior, 35, 45-62.

Shang C., 2015. The effect of smoke-free air law in bars on smoking initiation and relapse among teenagers and young adults. International Journal of Environmental Research and Public Health, 12, 504-520.

Skeer, M., Land, M.L., Cheng, D.M., Siegel, B., 2004. Smoking in Boston bars before and after a 100\% smoke-free regulation: an assessment of early compliance. Journal of Public Health Management \& Practice, 10, 501-507.

Song, A.V., Dutra, L.M., Neilands, T.B., Glantz, S.A., 2015. Association of smoke-free laws with lower percentages of new and current smokers among adolescents and young adults: an 11-year longitudinal study. JAMA Pediatrics 169, e152285.

Soteriades, E.S. DiFranza, J.R., 2003. Parents' socioeconomic status, adolescents' disposable income, and adolescents' smoking status in Massachusetts. American Journal of Public Health, 93, 1155-1160.

Stuber, J., Galea, S., Link, B.G., 2008. Smoking and the emergence of stigmatized social status. Social Science \& Medicine, 67, 420-430.

Swan, G.E., Javitz, H.S., Jack, L.M., Curry, S.J., McAfee, T., 2004. Heterogeneity in 12-month outcome among female and male smokers. Addiction, 99, 237-250. 
Tang, H., Cowling, D.W., Lloyd, J.C., Rogers, T., Koumjian, K.L., Stevens, C.M., Bal, D.G., 2003. Changes of attitudes and patronage behaviors in response to a smoke-free bar law. American Journal of Public Health, 93, 611-617.

Thomson, G., Wilson, N., Edwards., R., 2009. “At the frontier of tobacco control: a brief review of public attitudes toward smoke-free outdoor places." Nicotine \& Tobacco Research 11, 584-590.

Thomson, G., Wilson, N., Collins, D., Edwards, R., 2016. Attitudes to smoke-free outdoor regulations in the USA and Canada: a review of 89 surveys. Tobacco Control, 25, 506516.

Vuolo, M., Kadowaki, J., Kelly, B.C., 2016. A multilevel approach to constrained choices: the case of tobacco clean air restrictions. Journal of Health \& Social Behavior, 57, 351-372.

Wills, T.A., Resko, J.A., Ainette, M.G., Mendoza, D., 2004. Smoking onset in adolescence: a person-centered analysis with time-varying predictors. Health Psychology, 23, 158-167.

Yang, Y., 2010. Aging, cohorts, and methods. Pp. 17-30 in, Handbook of Aging and the Social Sciences, Seventh Edition, Binstock, R.H., George, L.K.(eds.). New York: Academic Press. 
Figure 1: Policy and denormalization, 2004-2011

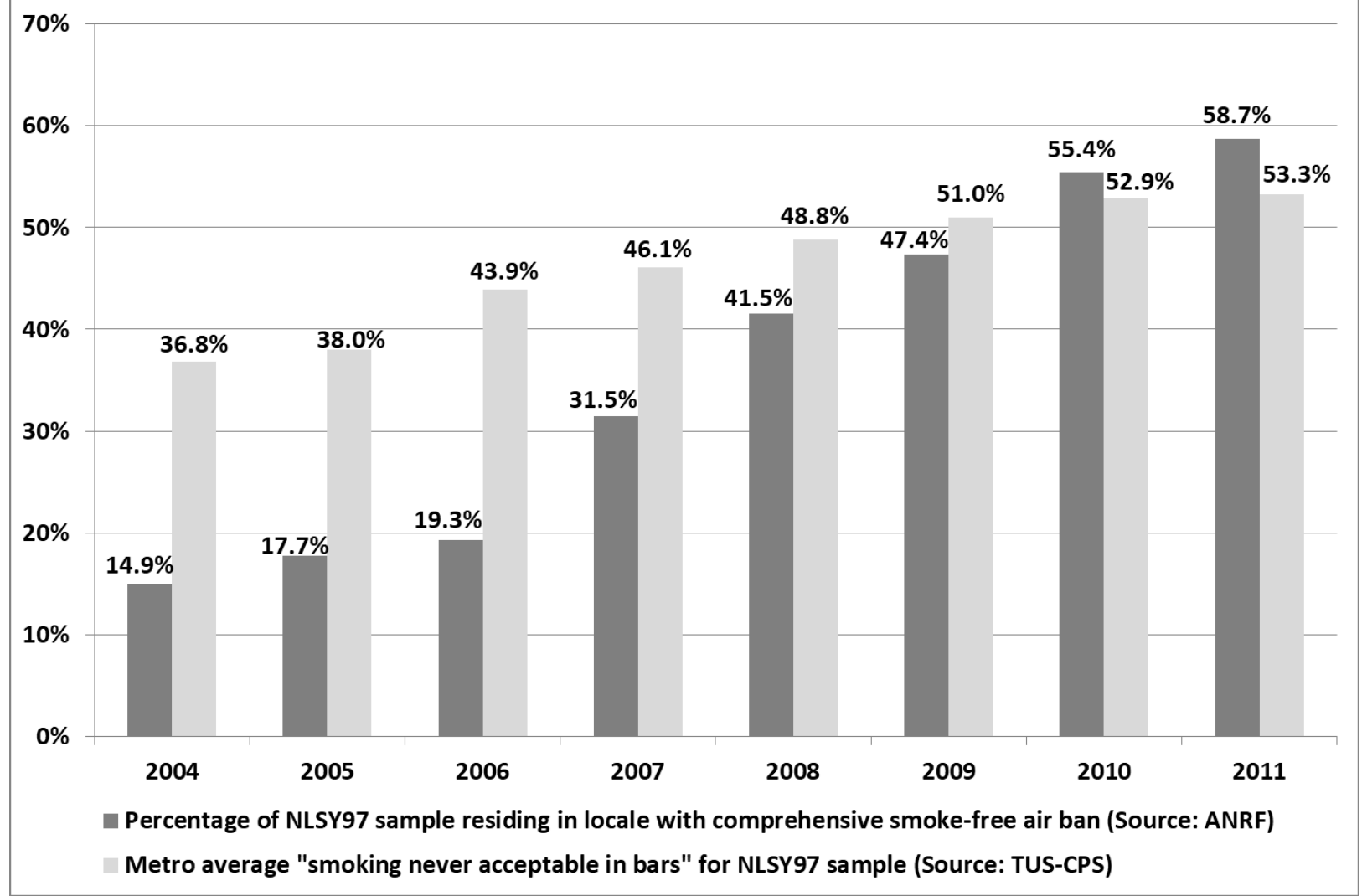


Table 1: Pooled Descriptive Statistics

\begin{tabular}{lc}
\hline & Percentage/ \\
Variable & Mean(SD) \\
\hline ANRF & \\
Comprehensive smoking ban & $42.98 \%$ \\
Youth possession restriction & $77.58 \%$ \\
Single cigarette sale restriction & $36.03 \%$ \\
Complete vending machine restriction & $11.15 \%$ \\
Any advertising restriction & $68.72 \%$ \\
Excise taxes(\$) & $1.36(1.14)$ \\
TUS-CPS Denormalization & \\
Percent supporting comprehensive ban & $47.84(13.25)$ \\
Census & \\
Population & \\
$<100,000$ & $10.69 \%$ \\
$100,000-250,000$ & $22.40 \%$ \\
$250,000-500,000$ & $14.46 \%$ \\
$500,000-1,000,000$ & $23.46 \%$ \\
$>1,000,000$ & $29.00 \%$ \\
Population density & $5,940.84(6,740.40)$ \\
Owner-occupied housing & $49.86(9.48)$ \\
Minors & $23.55(3.46)$ \\
Female-headed households & $11.95(3.54)$ \\
Non-Hispanic Whites & $46.03(18.74)$ \\
NLSY97 & \\
Past 30 days-any tobacco use & $32.84 \%$ \\
Past 30 days-smoked pack daily & $4.62 \%$ \\
Female & $51.55 \%$ \\
Race/Ethnicity & \\
White & $38.73 \%$ \\
Black & $34.34 \%$ \\
American Indian & $0.47 \%$ \\
Asian or Pacific Islander & $1.54 \%$ \\
Hispanic & $23.37 \%$ \\
Other & $1.55 \%$ \\
US native & $95.50 \%$ \\
Age & $25.37(2.53)$ \\
Age in 1997 & \\
12 & $20.89 \%$ \\
13 & $20.40 \%$ \\
14 & $19.94 \%$ \\
15 & $19.59 \%$ \\
16 & $19.18 \%$ \\
Age 17 past 30 day smoking & $30.11 \%$ \\
Parents' education & \\
Less than HS & $16.68 \%$ \\
$\quad$ \\
\hline
\end{tabular}


High school

Some college

Bachelor's

Parent health

Good-Excellent

Fair-poor

No info

Depression

HS grades: mostly A's

Peers smoking-1997

Almost none, $<10 \%$

About 25\%

About 50\%

About 75\%

Almost all, $>90 \%$

Living with parent

Education

HS dropout

HS/GED

Some college, not enrolled

Two-year degree

Four-year degree

Enrolled in HS

Enrolled in college

Moved between counties

Employment status

None

Part-time

Full-time

Job schedule

None

Day

Night

Irregular

Married

Parent
$29.71 \%$

$24.52 \%$

$29.09 \%$

$78.78 \%$

$13.30 \%$

$7.92 \%$

$4.54(2.45)$

$13.28 \%$

$28.31 \%$

$22.56 \%$

$23.83 \%$

$18.21 \%$

$7.09 \%$

$23.92 \%$

$10.20 \%$

$23.09 \%$

$23.29 \%$

$4.84 \%$

$24.97 \%$

$0.38 \%$

$13.25 \%$

$13.64 \%$

$24.58 \%$

$22.18 \%$

$53.24 \%$

$16.71 \%$

$54.01 \%$

$4.97 \%$

$24.31 \%$

$22.13 \%$

$40.39 \%$ 
Table 2: NLSY97 Multilevel Logistic Regression Models of Smoking on Smoking Ban and Denormalization

\begin{tabular}{|c|c|c|c|c|c|}
\hline & $\begin{array}{l}\text { Model 1: } \\
\text { Bivariate, no } \\
\text { controls } \\
\end{array}$ & $\begin{array}{l}\text { Model 2: Add } \\
\text { denormalization, no } \\
\text { controls }\end{array}$ & $\begin{array}{l}\text { Model 3: Add } \\
\text { denormalization } \\
\text { and controls }\end{array}$ & $\begin{array}{l}\text { Model 4: Add } \\
\text { interaction, no } \\
\text { controls }\end{array}$ & $\begin{array}{l}\text { Model 5: Add } \\
\text { interaction and } \\
\text { controls } \\
\end{array}$ \\
\hline & $\begin{array}{c}\text { OR } \\
(95 \% \mathrm{CI}) \\
\end{array}$ & $\begin{array}{c}\text { OR } \\
(95 \% \mathrm{CI}) \\
\end{array}$ & $\begin{array}{c}\text { OR } \\
(95 \% \mathrm{CI}) \\
\end{array}$ & $\begin{array}{c}\text { OR } \\
(95 \% \mathrm{CI}) \\
\end{array}$ & $\begin{array}{c}\text { OR } \\
(95 \% \mathrm{CI}) \\
\end{array}$ \\
\hline \multicolumn{6}{|l|}{ Any Past Month Smoking } \\
\hline smoking ban & $(0.420,0.677)$ & $(0.498,0.838)$ & $(0.582,0.983)$ & $(0.304,1.711)$ & $(0.223,1.234)$ \\
\hline $\begin{array}{l}\text { Denormalization of } \\
\text { smoking in bars }\end{array}$ & & $\begin{array}{c}0.838 * * * \\
(0.758,0.929)\end{array}$ & $\begin{array}{c}0.895^{*} \\
(0.807,0.992)\end{array}$ & $\begin{array}{c}0.846^{* *} \\
(0.750,0.954)\end{array}$ & $\begin{array}{c}0.870^{*} \\
(0.771,0.981)\end{array}$ \\
\hline Ban*Denormalization & & & & $\begin{array}{c}0.977 \\
(0.822,1.162) \\
\end{array}$ & $\begin{array}{c}1.080 \\
(0.910,1.282) \\
\end{array}$ \\
\hline \multicolumn{6}{|l|}{$\begin{array}{l}\text { Past Month Pack Per } \\
\text { Day }\end{array}$} \\
\hline $\begin{array}{l}\text { Comprehensive } \\
\text { smoking ban }\end{array}$ & $\begin{array}{c}0.572 * * \\
(0.381,0.860)\end{array}$ & $\begin{array}{c}0.739 \\
(0.477,1.144)\end{array}$ & $\begin{array}{c}0.664 \\
(0.422,1.046)\end{array}$ & $\begin{array}{c}0.548 \\
(0.112,2.684)\end{array}$ & $\begin{array}{c}0.273 \\
(0.059,1.266)\end{array}$ \\
\hline $\begin{array}{l}\text { Denormalization of } \\
\text { smoking in bars }\end{array}$ & & $\begin{array}{c}0.776^{* *} \\
(0.662,0.910)\end{array}$ & $\begin{array}{c}0.775^{* *} \\
(0.647,0.929)\end{array}$ & $\begin{array}{c}0.762 * * \\
(0.634,0.916)\end{array}$ & $\begin{array}{c}0.735^{* *} \\
(0.601,0.899)\end{array}$ \\
\hline Ban*Denormalization & & & & $\begin{array}{c}1.064 \\
(0.775,1.461) \\
\end{array}$ & $\begin{array}{c}1.211 \\
(0.885,1.657) \\
\end{array}$ \\
\hline
\end{tabular}

Note: Full models with controls in Appendices A\&B. 